\title{
Habituation and rehabituation of the monkey's orienting reflex as a function of stimulus variability
}

\author{
H. D. KIMMEL, M. S. RAICH, and A. F. BRENNAN \\ University of South Florida, Tampa, Florida 33620
}

\begin{abstract}
Fourteen Cebus albifrons monkeys were divided into two groups, one receiving an unchanging pure tone and the other a pure tone that varied in frequency from trial to trial. Following habituation of the skin conductance response, both groups were switched to a new tone for rehabituation. The monkeys needed significantly fewer trials to reach rehabituation than were needed in habituation, but the groups did not differ in this respect. Response magnitudes and reciprocals of response latency reduced significantly from habituation to rehabituation, but these effects also did not differ between groups. The group that received an unchanging pure tone showed significant reduction in response magnitude from the first to the second trials of habituation and rehabituation, but this reduction did not occur in the group that received frequency variability. It was concluded that the monkey's skin conductance response to pure tones habituates in a manner similar to that of the human, but that the retardation of habituation produced by stimulus variability in humans was not as strongly manifested in the monkey.
\end{abstract}

Reduced amplitude and increased latency of the skin conductance response (SCR) as a consequence of repeated administrations of an innocuous stimulus are the most commonly reported manifestations of orienting reflex (OR) habituation in humans (e.g., Sokolov, 1963). The electrodermal component of the OR in humans has been used as an index of the OR mainly because of its relative ease of measurement and the reliability of the effects of objectively defined independent variables, such as stimulus intensity and interstimulus interval, on its habituation rate (Geer, 1966; Sokolov, 1963). The susceptibility of SCR habituation to the influence of somewhat more subjective variables, such as uncertainty and stimulus variability (Kimmel, Piroch, \& Ray, 1979; Ray, Piroch, \& Kimmel, 1977), has added to this interest.

Systematic evaluation of the factors influencing SCR habituation in monkeys remains largely undone. The only published data on SCR habituation in the monkey were obtained with the rhesus preparation (Macaca mulatta) in connection with a series of studies of the effects of ablations of different brain regions on orienting reactions (Bagshaw, Kimble, \& Pribram, 1965; Kimble, Bagshaw, \& Pribram, 1965). The SCR of the cinnamon ringtail monkey (Cebus albifrons), the present experimental subject, has been under study for only a short time, in our laboratory (Kimmel \& Burns, 1977; Kimmel, Brennan,

This research was supported by a grant to the first author from the U.S. Army Medical Research and Development Command (DAMD 17-76-C-6053).
McLeod, Raich, \& Schonfeld, 1979), and no data on SCR habituation in the cebus preparation have been reported previously.

The effect of stimulus variability on habituation and rehabituation of the monkey's SCR was investigated in the present experiment. Based on our results with humans (Ray et al., 1977), it was hypothesized that monkeys receiving an initial series of repeated presentations of a pure tone of unchanging frequency would show more rapid SCR habituation (in terms of number of trials to criterion) than would monkeys that initially were given pure tones that varied in frequency unsystematically from trial to trial. Following the initial habituation series, all of the monkeys were rehabituated with an unchanging stimulus to determine whether any differences that might have developed during initial habituation would persist during rehabituation. Heart-rate data were collected, as well as the SCR.

\section{METHOD}

\section{Subjects}

Fourteen adult male Cebus albifrons monkeys served as subjects. These animals had been used about 1.5 years previously in a conditioning-extinction experiment involving visual stimuli and electric shocks (Kimmel, Brennan, et al., 1979), but none had ever experienced pure tones. The animals were divided randomly into two groups of seven.

\section{Apparatus}

The monkey was restrained during data collection in a specially designed Plexiglass chair with neck, waist, and chest yokes that prevented manual contact with the electrodes but 
permitted the monkey to remain comfortable for at least $1 \mathrm{~h}$. The monkey's tail protruded via a hole in the back of the chair. The chair also contained attachments for restraining the animal's feet after the SCR electrodes were attached. A bipolar SCR recording arrangement was used, with $1.2-\mathrm{cm}$ zinc-zinc sulfate electrodes in Teflon cups filled with saline electrode jelly. One of the Teflon cups was taped to the plantar surface of each foot. A constant voltage $(.5 \mathrm{Vdc})$ was applied to the two electrodes in series. The output of this constant-voltage circuit (Venables \& Christie, 1973) was proportional to skin conductance. This was amplified and fed into a voltage-to-frequency converter, the square-wave output of which was processed by a Data General Nova 2 digital computer. The computer was programmed to administer the stimuli and to search for SCRs from $1.0 \mathrm{sec}$ following stimulus onset to $5.0 \mathrm{sec}$ later, utilizing successive time bins of $.33 \mathrm{sec}$ and a response criterion of at least $.13 \mu \mathrm{mhos}$. Responses that met this criterion were counted and their amplitude and latency scored. Heart rate was picked up via two needle ECG electrodes that were attached subdermally to the right and left sides of the upper chest and taped in place. Heart rate was recorded on a Grass Model 7 polygraph.

The primate chair containing the monkey was placed inside a dimly lighted, sound-attenuated chamber $(86.7 \mathrm{~cm}$ wide, $73.7 \mathrm{~cm}$ deep, $86.7 \mathrm{~cm}$ high). A speaker behind the animal's head delivered continuous white noise at $70 \mathrm{~dB}$, physical reference. The tone stimuli were $80 \mathrm{~dB}$ in intensity, physical reference, and were presented via the same speaker, over the masking noise. Tone frequencies from 800 to $1,600 \mathrm{~Hz}$ were used, depending upon experimental condition, and tone duration was $5.0 \mathrm{sec}$. The stimuli were delivered at intervals ranging in 10-sec steps from 20 to $60 \mathrm{sec}$, with a mean of $40 \mathrm{sec}$, in a randomized sequence.

\section{Procedures}

Seven animals (fixed group) received a $1,200-\mathrm{Hz}$ tone repeatedly until two successive presentations occurred without a detectable SCR. For rehabituation, four of these animals were shifted to a $1,000-\mathrm{Hz}$ tone, and the other three to a $1,400-\mathrm{Hz}$ tone. Rehabituation continued until two successive nonresponses again occurred. The other seven monkeys (variable group) initially received $1,000,1,200$, and $1,400-\mathrm{Hz}$ tones in a random sequence, except that each frequency occurred once during the first three trials and none occurred more than twice successively thereafter. The variable group was also run to a criterion of two successive nonresponses during initial habituation. For rehabituation, four of these animals were shifted to a $1,600-\mathrm{Hz}$ tone and three to an $800-\mathrm{Hz}$ tone; a two-successive-nonresponse criterion was again used.

\section{RESULTS}

The average number of trials needed to reach criterion during habituation and rehabituation in the fixed and variable groups are shown in Table 1. It is clear that the predicted difference in habituation rate between the fixed and variable groups did not materialize. The only significant effect in the data shown in Table 1 was the overall reduction in number of trials from habituation $(\overline{\mathrm{X}}=6.07)$ to rehabituation $(\bar{X}=2.86)[F(1,12)=11.06, p<.01]$.

Because of the relatively rapid habituation that occurred, only the first two trials of habituation and rehabituation could be used for examination of the magnitude and latency measures. In this way, data from all 14 animals were available for analysis. Table 2 shows the average magnitude (microhmos)
Table 1

Average Number of Trials to Criterion in Habituation and Rehabituation for the Fixed and Variable Groups

\begin{tabular}{lcc}
\hline & Habituation & Rehabituation \\
\hline Fixed & 5.86 & 2.29 \\
Variable & 6.29 & 3.43 \\
\hline
\end{tabular}

Table 2

Average SCR Magnitude (Micromhos) and Reciprocal of Latency $(1 / \mathrm{sec})$ on Trials 1 and 2 of Habituation and Rehabituation for the Fixed and Variable Groups

\begin{tabular}{lrcccc}
\hline & \multicolumn{2}{c}{ Habituation } & \multicolumn{2}{c}{ Rehabituation } \\
\cline { 2 - 3 } & Trial 1 & Trial 2 & Trial 1 & Trial 2 \\
\hline Fixed & 1.52 & .71 & .28 & .00 \\
Variable & .90 & 1.14 & .51 & .57 \\
& \multicolumn{4}{c}{ Magnitude } \\
Fixed & .60 & Reciprocal of Latency \\
Variable & .56 & .55 & .34 & .17 \\
\hline
\end{tabular}

and the average reciprocal of latency $(1 / \mathrm{sec})$ of the SCR on the first two trials of habituation and rehabituation, for the fixed and variable groups. Reciprocals were used for the latency data so that the earliest possible scorable response, with a latency of $1.00 \mathrm{sec}$, received a score of 1.00 , while the latest possible response (i.e., a nonresponse), with an arbitrarily assigned latency of $6.00 \mathrm{sec}$, received a score of .17.

As can be seen in Table 2, SCR magnitudes and reciprocals of latency reduced from habituation to rehabituation. The SCR, in other words, reduced in size and occurred with increased delay, both of which are expected under conditions of repeated stimulation. Analysis of variance of the SCR magnitude measures showed that the overall reduction from habituation to rehabituation was significant $(F(1,12)=5.70, p<.05]$ but that the two groups did not differ significantly in this reduction. There was, however, a significant Groups by Trials interaction $[F(1,12)=7.91, p<.025]$, resulting from the fact that the fixed group's SCRs diminished from the first to the second trials of both habituation and rehabituation, while the variable group's SCRs were unchanged from the first to the second trials. Further analysis of the Groups by Trials interaction showed that it was separately significant in the habituation data $[\mathrm{F}(1,12)=8.83, \mathrm{p}<.025]$, but not in rehabituation. Analysis of variance of the latency data revealed that the only significant effect was the overall reduction in reciprocal of latency from habituation to rehabituation $[\mathrm{F}(1,12)=8.32, \mathrm{p}<$ $.025]$, but this decrease did not interact with groups. 
Six prestimulus interbeat intervals were used to establish an average baseline heart rate for each trial. Twenty-two poststimulus interbeat intervals were then used to generate an average heart-rate measure for 5 consecutive poststimulus seconds. The five poststimulus heart-rate measures for each trial were converted to change scores by subtracting the baseline measure for that trial from each of them. The heart-rate response showed no tendency to habituate over trials. Analysis of variance of the adjusted heart-rate measures revealed no significant effects other than the average course of heartrate change across successive poststimulus seconds $(F(4,48)=3.76, p<.05]$. As can be seen in Table 3, which shows the average heart rate change per poststimulus second, the heart-rate reaction following stimulus onset was decelerative, with some recovery beginning after about $3 \mathrm{sec}$. Trend analysis indicated that the downward linear component was significant $(\mathrm{F}(1,48)=4.59, \mathrm{p}<.05]$, as was the U-shaped quadratic component $[\mathrm{F}(1,48)=6.30, \mathrm{p}<.025]$.

\section{DISCUSSION}

Contrary to our prediction based upon results obtained with human subjects (Kimmel, Piroch, \& Ray, 1979; Ray et al., 1977), monkeys that received repeated presentations of an unchanging pure tone did not undergo SCR habituation more rapidly (i.e., in fewer trials) than animals that received tones that varied in frequency from trial to trial. Both groups of monkeys in the present study reached the habituation criterion in approximately six trials. Under equivalent conditions, human subjects also reach an SCR habituation criterion of two successive nonresponses in about six trials, but only when an unchanging stimulus is employed (Ray et al., 1977). The present failure to obtain a variability effect, such as is shown in human experiments, may be viewed as a failure of the variable group's experimental treatment to retard habituation relative to its base rate in the fixed group. This conclusion is based on the fact that both humans and monkeys reach a habituation criterion of two successive nonresponses in about six trials with an unchanging stimulus; the difference between the species occurs only when varying stimuli are used. This, of course, suggests that humans may be more sensitive to the changes in frequency of the pure tones used in this study than are the monkeys. This point will be pursued further below.

Even though there was no effect of stimulus variability in the trials-to-criterion data, SCR magnitude diminished from the first to the second trials of habituation and rehabituation in the fixed group but not in the variable group. This difference between the groups is consonant with the notion that stimulus
Table 3

Average Change in Heart Rate (in Beats per Minute) Following Onset of Auditory Stimulus

\begin{tabular}{lccccc}
\hline & \multicolumn{5}{c}{ Seconds Following Stimulus Onset } \\
\cline { 2 - 6 } & 1 & 2 & 3 & 4 & 5 \\
\hline Heart Rate Change & .34 & -.30 & -3.45 & -2.02 & -1.88 \\
\hline
\end{tabular}

variability retards habituation of the orienting reflex. It must be concluded that this effect was only weakly manifested in the present study, since it could be seen in the differential decline of SCR magnitudes in the fixed and variable groups but was not visible in either the trials-to-criterion or the latency data.

The present results do not provide a definitive basis for explaining the difference between humans and monkeys regarding the effect of stimulus variability on habituation. It is possible that humans may differ from monkeys in their sensitivity to the potential significance of variations in the frequency of sinusoidal tones. Stimulus variations of this type could sometimes be important in human day-to-day experience. Monkeys, on the other hand, have no experience at all with electronically produced sinusoidal tones, or with frequency variations of pure acoustic stimuli having potential adaptive significance. This line of speculation must be tempered, however, with the recognition that monkeys do communicate with sound and their SCR magnitude data did show a group difference, even though it was not translated into a difference in trials to criterion.

It is also possible that the use of the masking noise in the experimental chamber may have reduced the discriminability of the pure tones. Our previous studies with human subjects did not employ a masking noise, since in those studies the experimental IAC chamber was itself sufficiently sound attenuating to eliminate outside sounds. Again, however, the SCR magnitude differences that were observed indicate that frequency variations were registered by the animals.

Despite the foregoing limitations on the interpretation of the present results, the study appears to provide a useful starting point for the development of an organized body of knowledge regarding habituation of the electrodermal OR in the monkey. Decline in response strength and increase in latency clearly occurred as a consequence of stimulus repetition, transfer of habituation to new stimuli was shown, and some sensitivity to stimulus variability, similar to the effect seen previously in humans' SCRs, was suggested by the SCR magnitude data. Finally, the monkey's SCR appears to be quite similar qualitatively and quantitatively to that of the human, even in its basic rate of habituation under unchanging, repeated stimulation. 


\section{REFERENCES}

Bagshaw, M. H., Kimble, D. P., \& Pribram, K. H. The GSR of monkeys during orienting and habituation and after ablation of the amygdala, hippocampus and inferotemporal cortex. Neuropsychologia, 1965, 3, 111-119.

GeER, J. H. Effect of interstimulus intervals and rest-period length upon habituation of the orienting response. Journal of Experimental Psychology, 1966, 72, 617-619.

Kimble, D. P., Bagshaw, M. H., \& Pribram, K. H. The GSR of monkeys during orienting and habituation after selective ablations of the cingulate and frontal cortex. Neuropsychologia, 1965, 3, 121-128.

Kimmel, H. D., Brennan, A. F., McLeod, D. C., Raich, M. S., \& Schonfeld, L. I. Instrumental electrodermal conditioning in the monkey (Cebus albifrons): Acquisition and longterm retention. Animal Learning \& Behavior, 1979, in press.

KimmeL, H. D., \& BuRNS, R. A. Inter-effector influences in operant autonomic control. In J. Beatty \& H. Legewie (Eds.), Biofeedback and behavior. New York: Plenum, 1977.
Kimmel, H. D., Piroch, J., \& RAY, R. L. Monotony and uncertainty in the habituation of the orienting reflex. In H. D. Kimmel, E. H. van Olst, \& J. F. Orlebeke (Eds.), The orienting reflex in humans. Hillsdale, N.J: Erlbaum, 1979.

Ray, R. L., Piroch, J., \& Kimmel, H. D. The effect of task and stimulus variability on habituation of electrodermal and vasomotor reactions. Physiological Psychology, 1977, 5, 189-196.

Sokolov, Y. N. Perception and the conditioned reflex. Oxford: Pergamon Press, 1963.

Venables, P. H., \& Christie, M. J. Mechanisms, instrumentation, recording techniques, and quantification of responses. In W. F. Prokasy \& D. C. Raskin (Eds.), Electrodermal activity in psychological research. New York: Academic Press, 1973.

(Received for publication February 6, 1979; revision accepted July 20, 1979.) 